\title{
Mindfulness Training Increases Momentary Positive Emotions and Reward Experience in Adults Vulnerable to Depression: A Randomized Controlled Trial
}

Citation for published version (APA):

Geschwind, N., Peeters, F., Drukker, M., van Os, J., \& Wichers, M. (2011). Mindfulness Training Increases Momentary Positive Emotions and Reward Experience in Adults Vulnerable to Depression: A Randomized Controlled Trial. Journal of Consulting and Clinical Psychology, 79(5), 618-628. https://doi.org/10.1037/a0024595

Document status and date:

Published: 01/10/2011

DOI:

10.1037/a0024595

Document Version:

Publisher's PDF, also known as Version of record

Document license:

Taverne

Please check the document version of this publication:

- A submitted manuscript is the version of the article upon submission and before peer-review. There can be important differences between the submitted version and the official published version of record.

People interested in the research are advised to contact the author for the final version of the publication, or visit the DOI to the publisher's website.

- The final author version and the galley proof are versions of the publication after peer review.

- The final published version features the final layout of the paper including the volume, issue and page numbers.

Link to publication

\footnotetext{
General rights rights.

- You may freely distribute the URL identifying the publication in the public portal. please follow below link for the End User Agreement:

www.umlib.nl/taverne-license

Take down policy

If you believe that this document breaches copyright please contact us at:

repository@maastrichtuniversity.nl

providing details and we will investigate your claim.
}

Copyright and moral rights for the publications made accessible in the public portal are retained by the authors and/or other copyright owners and it is a condition of accessing publications that users recognise and abide by the legal requirements associated with these

- Users may download and print one copy of any publication from the public portal for the purpose of private study or research.

- You may not further distribute the material or use it for any profit-making activity or commercial gain

If the publication is distributed under the terms of Article 25fa of the Dutch Copyright Act, indicated by the "Taverne" license above, 


\title{
Mindfulness Training Increases Momentary Positive Emotions and Reward Experience in Adults Vulnerable to Depression: A Randomized Controlled Trial
}

\author{
Nicole Geschwind, Frenk Peeters, and \\ Marjan Drukker \\ Maastricht University Medical Centre
}

\author{
Jim van Os \\ Maastricht University Medical Centre and King's College \\ London
}

\author{
Marieke Wichers \\ Maastricht University Medical Centre
}

\begin{abstract}
Objective: To examine whether mindfulness-based cognitive therapy (MBCT) increases momentary positive emotions and the ability to make use of natural rewards in daily life. Method: Adults with a life-time history of depression and current residual depressive symptoms (mean age $=43.9$ years, $S D=$ 9.6; $75 \%$ female; all Caucasian) were randomized to MBCT $(n=64)$ or waitlist control (CONTROL; $n=66$ ) in a parallel, open-label, randomized controlled trial. The Experience Sampling Method was used to measure momentary positive emotions as well as appraisal of pleasant activities in daily life during 6 days before and after the intervention. Residual depressive symptoms were measured using the 17 -item Hamilton Depression Rating Scale (Hamilton, 1960). Results: MBCT compared to CONTROL was associated with significant increases in appraisals of positive emotion $\left(b^{*}=.39\right)$ and activity pleasantness $\left(b^{*}=.22\right)$ as well as enhanced ability to boost momentary positive emotions by engaging in pleasant activities $\left(b^{*}=.08\right.$; all $\left.p \mathrm{~s}<.005\right)$. Associations remained significant when corrected for reductions in depressive symptoms or for reductions in negative emotion, rumination, and worry. In the MBCT condition, increases in positive emotion variables were associated with reduction of residual depressive symptoms (all $p \mathrm{~s}<.05$ ). Conclusions: MBCT is associated with increased experience of momentary positive emotions as well as greater appreciation of, and enhanced responsiveness to, pleasant daily-life activities. These changes were unlikely to be pure epiphenomena of decreased depression and, given the role of positive emotions in resilience against depression, may contribute to the protective effects of MBCT against depressive relapse.
\end{abstract}

Keywords: MBCT, mindfulness-based cognitive therapy, residual symptoms, positive affect, ecological momentary assessment

Major depression is a common and highly recurrent clinical condition-an estimated $75 \%-90 \%$ of patients will have multiple episodes (e.g., Mueller et al., 1999). Even after different treatment steps, a considerable number of depressed participants continue to

This article was published Online First July 18, 2011.

Nicole Geschwind, Frenk Peeters, Marjan Drukker, and Marieke Wichers, European Graduate School for Neuroscience, SEARCH, Department of Psychiatry and Psychology, Maastricht University Medical Centre, Maastricht, the Netherlands; Jim van Os, European Graduate School for Neuroscience, SEARCH, Department of Psychiatry and Psychology, Maastricht University Medical Centre, and Department of Psychosis Studies, Institute of Psychiatry, King's College London, King's Health Partners, London, England.

Marieke Wichers was supported by the Dutch Organisation for Scientific Research (NWO, VENI Grant Nr. 916.76.147). We thank our study participants for their time and effort. Furthermore, we thank Rufa Diederen for her help with data collection, and Truda Driesen, Philippe Delespaul, Frieda van Goethem, and the 4D database team for their help with data entry.

Correspondence concerning this article should be addressed to Nicole Geschwind, Maastricht University Medical Centre, Vijverdalseweg 1, 6226 NB Maastricht, P.O. Box 616 (VIJV-SN2), 6200 MD Maastricht, the Netherlands. E-mail: n.geschwind@maastrichtuniversity.nl experience residual symptoms of depression (Rush et al., 2006). Residual symptoms not only impact negatively on quality of life, they are also associated with three times faster relapse, compared to full recovery (Judd et al., 1998; Rush et al., 2006). Residual symptoms commonly include sleep disturbances; loss of energy; and decreased pleasure, motivation, and interest. Overall, this pattern of symptoms is consistent with lower levels of positive affect (PA; Nutt et al., 2007; Watson, Clark, \& Carey, 1988)—a core symptom of major depressive disorder. Previous studies suggest that PA promotes prevention of and recovery from depression (Geschwind et al., 2011; Morris, Bylsma, \& Rottenberg, 2009; Wichers et al., 2010). Prevention efforts focused on increases in PA are therefore likely to (a) improve residual symptoms and well-being and (b) prevent relapse.

\section{PA, Well-Being, and Depression}

Experiencing positive emotions and having a positive attitude have beneficial effects on mental and physical health in general (Seligman, Steen, \& Peterson, 2005). A meta-analysis of crosssectional, longitudinal, and experimental studies demonstrated that PA was associated with and preceded success, indicating that PA 
facilitates accomplishment (Lyubomirsky, King, \& Diener, 2005). Evidence also suggests that PA is a source of resilience against pain and negative affectivity. For example, in a study in which women with chronic pain were interviewed weekly, higher levels of PA predicted lower levels of pain in subsequent weeks. Higher weekly levels of PA also resulted in lower levels of negative affect (NA) both directly and in interaction with stress and pain (Zautra, Johnson, \& Davis, 2005). A recent study suggests that especially in-the-moment positive emotions, rather than more general satisfaction with life, are associated with increased resilience (Cohn, Fredrickson, Brown, Mikels, \& Conway, 2009). Momentary assessment studies, in which experiences are sampled repeatedly at random moments in daily life, support this view. For example, high daily life reward experience (i.e., the ability to generate PA from pleasant daily events) predicted increased resilience against later affective symptoms in participants vulnerable to depression (Geschwind et al., 2010; Wichers et al., 2010). Furthermore, in participants with a lifetime history of depression, scoring one standard deviation higher on the ability to generate PA from pleasant daily life events was associated with a three-fold reduction in risk to experience a future episode (Wichers et al., 2010).

Studies show that depressed participants, compared to neverdepressed controls, experience less PA in the course of daily life (Barge-Schaapveld, Nicolson, Berkhof, \& deVries, 1999; Bylsma, Taylor-Clift, \& Rottenberg, 2011; Peeters, Berkhof, Delespaul, Rottenberg, \& Nicolson, 2006). Also, they generate less PA from pleasant stimuli during experimental tasks (Bylsma, Morris, \& Rottenberg, 2008), though in daily life this phenomenon is debatable (Bylsma et al., 2011; Peeters, Nicolson, Berkhof, Delespaul, \& De Vries, 2003)

Taken together, the evidence suggests that (a) high levels of PA have beneficial effects on vulnerability to, prevention of, and recovery from depression and (b) depressed individuals generate less PA compared to nondepressed individuals. In other words, PA may represent a resilience phenotype against depression. Individuals vulnerable to depression may therefore benefit from learning to experience more PA. The question arises how people can reshape emotional processes in a way that heightens their ability to experience PA in their daily lives. One momentary assessment study showed that response to a 6-week treatment with antidepressant medication was associated with a heightened ability to boost PA through pleasant activities, relative to baseline (Wichers et al., 2009). However, advocating use of antidepressant medication for prevention purposes only is problematic - and whether a behavioral, nonpharmacological intervention can be used to heighten participants' ability to generate positive emotions in daily life has never been tested.

An intervention currently receiving empirical support for the prevention of depressive relapse and recurrence, and for the treatment of residual depressive symptoms, is mindfulness-based cognitive therapy (MBCT; Segal, Williams, \& Teasdale, 2002). Although many authors assume changes in cognitive processes to be a core element of the beneficial effects of MBCT (Segal et al., 2002), it is unclear whether and how MBCT affects alterations in emotional experiences. As we argue below, there is reason to believe that MBCT may have the potential to induce changes in resilience phenotypes associated with PA.

\section{MBCT}

During mindfulness exercises, participants attempt to maintain attention on a particular focus, for example, their own breathing. Whenever the attention wanders away from breathing to thoughts or feelings, participants are encouraged to acknowledge and accept these thoughts and feelings but then to let go of them and to direct their attention back to breathing. Participants then use the same approach in the course of daily life: They aim to focus on the "here and now" (to engage with the present experience) and to redirect their attention whenever they notice that it has switched to distracting thoughts and worries. Next to this attentional aspect, mindfulness also works on an attitudinal dimension by promoting the cultivation of an open, curious, and mild orientation of mind. The essence of a mindful state is to be fully in the present moment, to experience the present moment without judgment or evaluation and without worrying about the future or ruminating about past experiences (Kabat-Zinn, 1990).

MBCT is specifically designed to prevent depressive relapse and recurrence. MBCT combines methods of meditation and mindfulness training (Kabat-Zinn, 1990) with features of cognitive therapy for depression (Beck, Rush, Shaw, \& Emergy, 1979; Teasdale, Segal, \& Williams, 1995). The original idea behind MBCT was to train participants to disengage from automatic negative thinking patterns that arise during dysphoric mood and facilitate relapse (Teasdale et al., 2000). Several studies have shown that MBCT is associated with reduced depressive symptoms and lower risk of relapse (Bondolfi et al., 2010; Kuyken et al., 2008; Ma \& Teasdale, 2004; Teasdale et al., 2000). Furthermore, recent studies found that MBCT is associated with reduced cognitive reactivity (Kuyken et al., 2010; Raes, Dewulf, Van Heeringen, \& Williams, 2009), rumination (Williams, 2008), and worry (Evans et al., 2008).

However, there is reason to believe that MBCT might also increase participants' capacity for the experience of PA (Geschwind et al., 2010; see also Garland et al., 2010). Studies show that more advanced meditators experience more positive emotions (Easterlin \& Cardena, 1998-1999) and that people report more positive emotions when in a mindful compared to a nonmindful state (Brown \& Ryan, 2003; Killingsworth \& Gilbert, 2010). Furthermore, in a randomized controlled trial, participants of loving-kindness meditation reported stronger increases in PA over time compared to control participants (Fredrickson, Cohn, Coffey, Pek, \& Finkel, 2008). During loving-kindness meditation, participants practice to experience love and compassion first toward themselves and then toward loved ones, acquaintances, and strangers (Fredrickson et al., 2008). This very explicit focus on positive emotions may, however, create a demand bias, which is much less pronounced in MBCT. Although awareness of pleasant events and nourishing activities is addressed during two to three of the eight training sessions (just as reactions to stressful situations are dealt with), the main focus during MBCT sessions is to develop an increased moment-to-moment awareness of experience (Baer, 2003). As pleasant events and emotions are usually less enduring, intense, and attention-grabbing than unpleasant events and emotions (Baumeister, Bratslavsky, Finkenauer, \& Vohs, 2001), they may be more easily overlooked than their unpleasant counterparts. Increased moment-to-moment awareness, achieved in a mindful state, may help people to perceive fleeting pleasant events or 
emotions and to generate more enjoyment from pleasant activities (Schroevers \& Brandsma, 2010).

\section{Hypotheses}

PA is associated with increased resilience against depression, but it remains unclear to which extent phenotypes related to PA can be modified trough behavioral interventions. We propose that MBCT is an intervention that increases the ability to make use of natural, moment-to-moment rewards in the environment, thereby increasing PA. Our primary hypothesis is that, after MBCT, participants (a) experience more PA, (b) rate pleasant daily-life activities as more pleasant, and (c) experience more reward (i.e., generate more PA from engaging in pleasant activities). Because previous literature has shown that MBCT is associated with decreased worry, rumination (see above), and NA (Schroevers \& Brandsma, 2010), we additionally examine to which extent potential changes in PA, pleasant activities, and reward experience are independent of decreases in worry, rumination, and NA. Because of the protective links between PA and depression, a secondary hypothesis is that increases in PA, pleasant activities, and reward experience will be associated with decreases in depressive symptoms within the MBCT condition.

Hypotheses were tested in an open-label, parallel, randomized controlled trial comparing participants who continued with treatment as usual to participants who, additionally, received MBCT. To measure the fleeting momentary experience of positive emotions (Garland et al., 2010) in an ecologically valid and reliable manner, the Experience Sampling Method (ESM) was used. ESM is a momentary assessment technique in which participants are prompted to report on their current experiences at random moments during the day. ESM is therefore ideally suited to investigate changes in people's emotional reactions to their daily environment (Csikszentmihalyi \& Larson, 1987). To our knowledge, this is the first study to examine how MBCT is associated with changes in people's momentary affective responses in their daily life context. Furthermore, this trial is the first to examine whether a nonpharmacological intervention can be used to modify a resilience phenotype (the ability to experience and generate positive emotions in daily life) in a sample vulnerable to depression.

\section{Method}

\section{Participant Characteristics}

For the current study (acronym: MindMaastricht; trial number: NTR1084, Netherlands Trial Register), adults with residual symptomatology after at least one episode of major depressive disorder were recruited from outpatient mental health care facilities in Maastricht (the Netherlands) and through posters in public spaces. Residual symptoms are associated with higher risk of relapse (Judd et al., 1998) and were therefore required as an indicator of vulnerability to depression. Residual symptoms were defined as a score of seven or higher on the 17-item Hamilton Depression Rating Scale (HDRS; Hamilton, 1960) at the time of screening. Exclusion criteria included the following: fulfilling criteria for a current depressive episode, schizophrenia, or psychotic episodes in the past year, and recent (past 4 weeks) or upcoming changes in ongoing psychological or pharmacological treatment. Currently depressed individuals were excluded because, at trial preparation, there was no evidence that currently depressed individuals were able to participate in or benefit from MBCT. Relevant sociodemographic and clinical characteristics are displayed in Table 1.

\section{Sampling Procedures}

All study procedures were approved by the Medical Ethics Committee of Maastricht University Medical Centre, and all participants signed an informed consent form. An initial screening of potential participants for this randomized controlled trial was performed by phone to check for availability during the study period and likelihood of meeting inclusion and exclusion criteria. A second screening included administration of the Structured Clinical Interview for DSM IV-Axis I (First, Spitzer, Gibbon, \& Williams, 2002) and the 17-item HDRS by trained psychologists. Eligible participants were invited for a detailed one-on-one explanation of the experience sampling procedure, and then they took part in the baseline assessment. The baseline assessment consisted of 6 days of experience sampling in their own environment (see the ESM section) and subsequent administration of a battery of questionnaires (see the Measures section) as well as the HDRS interview (in the laboratory). After the baseline assessment, participants were randomized to either MBCT or waitlist control (CONTROL; allocation ratio $1: 1$ ) if they were likely to have at least 20 valid ESM assessments (Delespaul, 1995; see the ESM section). After either 8 weeks of MBCT (see the Intervention section) or equivalent waiting time (in the CONTROL condition), participants again took part in 6 days of experience sampling, followed by the administration of the HDRS and the questionnaires. All participants were compensated with gift vouchers worth 50 Euros. Participants in the CONTROL condition had the opportunity to take part in MBCT after the postintervention assessment.

Randomization to treatment condition was stratified according to number of depressive episodes (two or less vs. three or more), as previous studies suggest a greater benefit for those with three or more previous episodes (Ma \& Teasdale, 2004; Teasdale et al., 2000). An independent researcher not involved in the project generated the randomization sequence in blocks of five (using the

Table 1

Baseline Demographic and Clinical Characteristics per Group

\begin{tabular}{lcc}
\hline \multicolumn{1}{c}{ Variable } & $\begin{array}{c}\text { MBCT } \\
(n=63)\end{array}$ & $\begin{array}{c}\text { CONTROL } \\
(n=66)\end{array}$ \\
\hline Age $(M, S D)$ & $44.6(9.7)$ & $43.2(9.5)$ \\
Female gender (\%) & 79 & 73 \\
Full-/part-time work (\%) & 62 & 68 \\
Illness/unemployment benefits (\%) & 19 & 23 \\
Living with partner/own family (\%) & 64 & 64 \\
$\leq 2$ previous episodes of MDD (\%) & 56 & 55 \\
$\geq 3$ previous episodes of MDD (\%) & 44 & 45 \\
Comorbid anxiety disorder (present) $(\%)$ & 35 & 49 \\
Comorbid anxiety disorder (past) $(\%)$ & 51 & 64 \\
Current psycho-counseling/-therapy (\%) & 13 & 12 \\
Current use of antidepressants (\%) & 32 & 38 \\
(Occasional) use of benzodiazepines (\%) & 8 & 8 \\
\hline
\end{tabular}

Note. There were no significant differences between groups (at $p<.05$ ) $\mathrm{MBCT}=$ mindfulness-based cognitive therapy; CONTROL $=$ waitlist control condition; MDD = major depressive disorder. 
sequence generator on www.random.org) and wrote the randomization code into sealed numbered envelopes. After completion of all baseline assessments, the researcher allocated participants to their treatment condition based on the randomization code in the sealed envelope (opened in order of sequence). No masking of treatment condition took place.

\section{Sample Size and Power}

Sample size $(n \geq 120)$ was determined on the basis of sufficient power for gene-environment interactions (not analyzed here). Post hoc power calculations for the current analyses (using Stata 11s SAMPSI command; StataCorp, 2009) indicated a power of $>.90$ to detect small effects $(d=0.2)$ in the parameter of interest: the Group $\times$ Time interaction.

\section{Intervention (MBCT)}

Content of MBCT training sessions followed the protocol of Segal et al. (2002). Trainings consisted of eight weekly meetings lasting $2.5 \mathrm{hr}$ and were run in groups of $10-15$ participants (thus occasionally larger than the usual 10-12 participants per group). Assessment periods for control participants were matched to those of MBCT participants. Sessions included guided meditation, experiential exercises, and discussions. In addition to the weekly group sessions, participants received CDs with guided exercises and were assigned daily homework exercises (30-60 min daily). Trainings were given by experienced trainers in a center specialized in mindfulness trainings. All trainers were supervised by an experienced health care professional who had trained with Teasdale and Williams, the co-developers of MBCT (Teasdale et al., 1995).

\section{ESM}

ESM is a momentary assessment method to assess participants in their daily living environment, thus providing repeated in-themoment assessments of affect in a prospective and ecologically valid manner (Csikszentmihalyi \& Larson, 1987; Peeters et al., 2003). Compared to retrospective questionnaires and interviews, ESM offers several advantages: (a) enhanced ecological validity, because participants are assessed in their normal daily environments; (b) minimized retrospective bias, because participants' experiences are assessed in the moment; and (c) enhanced reliability, because participants' experiences are assessed repeatedly (Csikszentmihalyi \& Larson, 1987).

In the current study, participants received a digital wristwatch and a set of ESM self-assessment forms collated in a booklet for each day. The wristwatch was programmed to emit a signal ("beep") at an unpredictable moment in each of ten 90-min time blocks between 7:30 a.m. and 10:30 p.m., on 6 consecutive days, resulting in a maximum of 60 beeps per study period. After each beep, participants were asked to fill out the ESM self-assessment forms previously handed to them, collecting reports of current mood and context. All self-assessments were rated on 7-point Likert scales. Trained research assistants explained the ESM procedure to the participants during an initial briefing session, and a practice form was completed to confirm that participants understood the 7-point Likert scale. Participants were instructed to complete their reports immediately after the beep, thus minimizing memory distortion, and to record the time at which they completed the form. All reports not filled in within $15 \mathrm{~min}$ after the actual beep were excluded from the analysis, because previous work (Delespaul, 1995) has shown that reports completed after this interval are less reliable and consequently less valid. For the same reason, participants with less than 20 valid reports at baseline were excluded from the analysis (Delespaul, 1995).

\section{Measures}

Pleasantness of daily life activities. To define pleasantness of daily life activities in an ecologically valid manner, ESM self-rated appraisals of ongoing activities were used, consistent with several previous studies of emotional reactivity to appraised daily activities and contexts (Myin-Germeys \& van Os, 2007; Myin-Germeys, van Os, Schwartz, Stone, \& Delespaul, 2001; Wichers et al., 2009). Participants rated their current activity on a 7-point Likert scale $(1=$ not at all, and $7=$ very $)$. Factor analysis supported inclusion of four items for activity appraisal (with factor loadings > .6), namely, "I enjoy this activity," "This activity requires effort," "I would prefer to do something else," and "I am skilled at doing this activity." Two items ("I feel I'm being active," and "This is a challenge") had low factor loadings (.05 and .12, respectively) and were consequently not included in the activity pleasantness score. On the basis of the included ratings, a variable reflecting "pleasantness of current activity" was generated. Before creating the activity pleasantness variable, the items "This activity requires effort," and "I would prefer to do something else" were first recoded so that high scores reflected lower appraised effort and higher preference for the current activity. Consistent with Wichers et al. (2009), low scores $(\leq 4)$ on all four items were set to zero (so that negative activity appraisals did not contribute to the overall score), and higher scores were recoded $(5=1,6=2,7=$ 3) before calculating a sum score for activity pleasantness. High pleasantness thus reflected high skill, low effort, low preference for doing something else, and high enjoyment of the activity.

PA and NA. At each beep, several ESM mood adjectives were assessed on 7-point Likert scales ranging from 1 (not at all) to 7 (very). Consistent with previous work (Myin-Germeys et al., 2001; Wichers et al., 2010), principal component factor analysis with oblique rotation was used to generate a factor representing PA and a factor representing NA. The mood adjectives "happy," "satisfied," "strong," "enthusiastic," "curious," "animated," and "inspired" loaded on the PA factor $(\alpha=.89)$, whereas "down," "anxious," "lonely," "suspicious," "disappointed," "insecure," and "guilty" loaded on the NA factor $(\alpha=.86)$. One mood item ("I feel relaxed") was not included in the PA factor due to low factor loadings $(<.6)$. Mean levels of PA and NA were then computed per participant and beep moment.

Reward experience. Reward experience was conceptualized as the effect (the standardized coefficient $b^{*}$ ) of pleasant activities on momentary PA. This coefficient captures the increase in PA when engaging in pleasant activities, relative to baseline. Reward experience thus was not a precalculated variable but was hidden in the outcome of the analyses (consistent with Wichers et al., 2009), so there was no way in which participants could consciously fake the outcome on this variable. 
HDRS. The 17-item HDRS (Hamilton, 1960) was administered by two trained research assistants with master's degrees in psychology. The HDRS is a semistructured interview designed to assess depressive symptoms over the past week. It is one of the most often used rating scales in depression research, and internal, interrater, and retest reliability estimates for the overall HDRS are good (Bagby, Ryder, Schuller, \& Marshall, 2004). Only the overall score was used for the analyses, and interrater reliability for the total score was high (intraclass correlation coefficient $=.97$ ). To provide information on interrater reliability, both interviewers had independently rated eight videotaped HDRS interviews with patients varying in strength of residual depressive symptoms.

Inventory of Depressive Symptoms, Self-Rating (IDS-SR). The IDS-SR (Rush, Gullion, Basco, Jarrett, \& Trivedi, 1996) is a self-rated scale, which includes 30 items rated on a scale from 0 to 3. Because the rating of "appetite" and "weight" is duplicated (separate items for increases and decreases), only 28 items are taken into account for the final score. The scale is sensitive to change and has good psychometric properties (Rush et al., 1996). Internal consistency in the current sample was .85.

Penn State Worry Questionnaire (PSWQ). A Dutch version of the 16-item PSWQ (Meyer, Miller, Metzger, \& Borkovec, 1990) was used to assess worrying. The PSWQ emerged from factor analysis of a large number of items and was found to possess high internal consistency and good test-retest reliability (Meyer et al., 1990). Internal consistency in our sample was .90 .

Rumination on Sadness Scale (RSS). The approved Dutch back-translation (Raes, Hermans, \& Eelen, 2003) of the RSS (Conway, Csank, Holm, \& Blake, 2000) was used to measure rumination. The RSS contains 13 items comprising one factor, has an adequate test-retest stability over a 2- to 3-week period, and has good convergent and discriminant validity (Conway et al., 2000). Internal consistency in the current sample was .90 , similar to earlier studies (e.g., Conway et al., 2000).

\section{Statistical Methods}

ESM data have a hierarchical structure. Thus, multiple observations (Level 1) are clustered within participants (Level 2). Multilevel analyses take the variability associated with each level of nesting into account (Snijders \& Bosker, 1999). Multilevel linear regression analyses, using the XTREG command in STATA 11.1 (StataCorp, 2009), were applied to the ESM data. "Reward Experience" was conceptualized as the effect of positively appraised daily life activities on PA (i.e., the boost in PA when participants engage in more pleasant compared to neutral activities).

First, we examined the effects of MBCT on change in PA, pleasantness of activities, and reward experience, relative to CONTROL. Thus, in the models of PA and activity pleasantness, the two-way interaction between time (baseline vs. post assessment) and treatment group (CONTROL vs. MBCT) was the parameter of interest. For reward experience, the three-way interaction between time (baseline vs. post assessment), treatment group (CONTROL vs. MBCT), and activity pleasantness in the model of PA was the parameter of interest. The MARGINS, DYDX command (StataCorp, 2009) was used to calculate the effect of activity pleasantness on PA in the four different conditions (MBCT and CONTROL, at baseline and postintervention). Per treatment group, significance of differences between baseline and postas- sessment were then assessed with Stata's TEST command, which uses the Wald test (Clayton \& Hill, 1993). To refute concerns that improvements in PA-related variables may simply reflect epiphenomena of decreased depression scores, analyses were re-run post hoc while correcting for reduction on the HDRS.

Second, to examine to what extent the effect of treatment on PA, pleasant activities, and reward experience was independent of changes in third variables known from MBCT research, the abovementioned analyses were repeated while including the interaction terms containing changes in worry, rumination, and NA (i.e., Time $\times$ Worry + Time $\times$ Rumination + Time $\times$ NA).

Third, we examined to what extent MBCT-related improvements in the variables PA, activity pleasantness, and reward experience were associated with decreases in depressive symptoms. For this reason, analyses for all three variables were repeated in the MBCT group only, using "Reduction on the HDRS" (baseline minus post assessment) in the interaction terms instead of treatment group. To visualize these associations more clearly, and to examine dose-response associations between improvements in PA-related variables and depressive symptoms, we then divided improvement on the HDRS into tertiles. The strength of associations was tested using the margins command, and significance was assessed using the Wald test (Clayton \& Hill, 1993). To ensure that these associations were independent of the format of measurement, the analyses were repeated using the self-report IDS-SR to classify change in depressive symptoms (instead of the clinician-rated HDRS).

Because average levels at baseline naturally influence the extent to which a variable can increase (e.g., ceiling effects), the analyses on PA and reward experience were corrected for individually averaged baseline levels of PA. Similarly, the analyses on activity pleasantness were corrected for averaged baseline ratings of activity pleasantness.

The reported analyses are based on the whole sample (intention to treat). Participants who attended less than four MBCT sessions $(n=3)$ were excluded for the per-protocol analysis (outcomes were similar and are not reported in detail).

\section{Results}

\section{Participants}

Recruitment of participants started in January 2008 and ended in February 2009, and all postintervention assessments were completed by August 2009, when the predetermined number of participants was reached. Sociodemographic and clinical characteristics of MBCT and CONTROL participants are displayed in Table 1. At baseline, there were no large or significant differences between treatment groups with respect to sociodemographic and clinical characteristics. Table 2 shows baseline and postassessment scores of variables used in the analyses, stratified by treatment group. Again, there were no large or significant differences between groups at baseline. Participant flow through the study is displayed in Figure 1. No known harms or unintended treatment effects were reported in either group.

Participants completed 12,453 entries in total. Of these, 559 (4\%) were excluded as invalid entries, because completion times fell outside the predetermined window of $15 \mathrm{~min}$ after the beep. On average, participants completed 49 (of $60 ; S D=7.6$ ) valid entries 
Table 2

Means and Standard Deviations of Variables Used in the Analyses, Stratified by Group and Measurement Occasion

\begin{tabular}{|c|c|c|c|c|}
\hline \multirow[b]{3}{*}{ Variable } & \multicolumn{2}{|c|}{$\operatorname{MBCT}(n=63)$} & \multicolumn{2}{|c|}{ CONTROL $(n=66)$} \\
\hline & Baseline & Post & Baseline & Post \\
\hline & $M(S D)$ & $M(S D)$ & $M(S D)$ & $M(S D)$ \\
\hline PA & $3.7(1.3)$ & $4.2(1.3)_{\mathrm{b}}$ & $3.9(1.2)$ & $3.9(1.2)$ \\
\hline Pleasantness & $2.3(1.3)_{\mathrm{a}}$ & $2.5(1.3)_{\mathrm{b}}$ & $2.3(1.3)_{a}$ & $2.1(1.3)_{\mathrm{c}}$ \\
\hline HDRS & $10.3(3.7)_{\mathrm{a}}^{\mathrm{a}}$ & $7.1(4.8)_{\mathrm{b}}$ & $10.2(3.5)_{\mathrm{a}}$ & $9.7(4.0)_{\mathrm{a}}$ \\
\hline IDS-SR & $22.3(10.7)$ & $14.6(10.7)_{\mathrm{b}}$ & $22.5(8.7)$ & $19.2(9.5)$ \\
\hline RSS & $42.2(9.7)_{a}$ & $34.4(9.8)_{\mathrm{b}}$ & $40.8(9.7)_{a}$ & $37.9(10.0)_{\mathrm{a}}$ \\
\hline PSWQ & $59.7(10.9)_{\mathrm{a}}$ & $50.6(11.5)_{\mathrm{b}}$ & $59.7(10.1)_{\mathrm{a}}$ & $56.3(10.3)_{\mathrm{a}}$ \\
\hline NA & $2.0(1.1)_{\mathrm{a}}$ & $1.6(.8)_{\mathrm{b}}$ & $2.0(1.0)_{\mathrm{a}}$ & $2.0(1.0)_{\mathrm{a}}$ \\
\hline MBCT sessions attended & & $7.2(1.5)$ & & \\
\hline Minutes practiced per day & & $29.7(13.2)$ & & \\
\hline
\end{tabular}

Note. Per row, variables sharing the same subscript do not differ at $p<.05$. MBCT $=$ mindfulness-based cognitive therapy; $\mathrm{CONTROL}=$ waitlist control condition; $\mathrm{PA}=$ positive affect; Pleasantness $=$ appraisal of activity pleasantness; HDRS $=$ Hamilton Depression Rating Scale; IDS-SR = Inventory of Depressive Symptoms, Self-Rating; RSS = Rumination on Sadness Scale; PSWQ = Penn State Worry Questionnaire; $\mathrm{NA}=$ negative affect.

per assessment period. One participant had completed fewer than 20 valid entries at baseline and was therefore excluded from the analyses.

\section{Effects of MBCT on PA-Related Variables}

MBCT compared to CONTROL was associated with significant increases in PA, $b^{*}=.40,95 \%$ CI $[.33, .46], \chi^{2}(1)=163.02, p<$ .001. Similarly, MBCT was associated with increases in activity pleasantness, compared to CONTROL, $b^{*}=.22,95 \%$ CI $[.15$, $.29], \chi^{2}(1)=39.09, p<.001$. Also, reward experience increased significantly in the MBCT group, compared to CONTROL, $b^{*}=$ $.08,95 \%$ CI $[.03, .14], \chi^{2}(1)=8.23, p=.004$. Standardized predicted values of PA-related variables, by group and assessment period, are displayed in Figure 2. Effects of treatment condition on PA-related variables were similar when the analyses were repeated while correcting for change in residual depressive symptoms $\left(b^{*}=\right.$ $.40, .22$, and .08 , for PA, activity pleasantness, and reward experience, respectively), indicating that the effects of $\mathrm{MBCT}$ on PA-related variables were not just epiphenomena of reduction in depressive symptoms.

\section{Independence of Effects of MBCT on PA-Related Variables}

As can be seen in Table 2, MBCT was also associated with significant reductions in worry, rumination, and NA compared to CONTROL. However, effects of MBCT on PA-related variables were reduced but remained evident and statistically significant when changes in worry, rumination, and NA were included in the analyses. The effect size of the Time (baseline vs. postassessment) $\times$ Group (MBCT vs. CONTROL) interaction term then became $b^{*}=.19, p<.001$ (unadjusted $b^{*}=.40$ ) in the model of PA, and $b^{*}=.16, p<.001$ (unadjusted $b^{*}=.22$ ) in the model of activity pleasantness. In the model of reward experience, the effect size of the three-way interaction Time $\times$ Group $\times$ Activity Pleasantness became $b^{*}=.07, p=.007$ (unadjusted $b^{*}=.08$ ).

\section{Clinical Significance: Association Between Symptom Reduction and PA-Related Increases}

With regard to clinical significance, analyses restricted to the MBCT group showed that reduction on the HDRS was associated with significant increases in PA $\left(b^{*}=.30,95 \%\right.$ CI $[.26, .34], p=$ $.000)$, activity pleasantness $\left(b^{*}=.05,95 \%\right.$ CI $\left.[.002, .10], p=.000\right)$, and reward experience $\left(b^{*}=.06,95 \%\right.$ CI $\left.[.02, .09], p=.003\right) .{ }^{1}$ To examine dose-response relationships, participants were classified as low $(n=21$, mean reduction in HDRS scores $\Delta M=-1.94, S D=$ 2.83 , range $=-9$ to 1$)$, medium $(n=22$, HDRS $\Delta M=3.89, S D=$ 1.02 , range $=2-5)$, and high $(n=20$, HDRS $\Delta M=8.31, S D=1.53$, range $=6-12$ ) responders to $\mathrm{MBCT}$, using a tertile split. Figure 3 displays standardized predicted values of PA-related variables by tertiles of response and assessment period (pre- vs. post-MBCT), confirming the predicted pattern of results. Results were similar when the IDS-SR was used to assess symptom reduction instead of the HDRS.

\section{Discussion}

\section{Current Findings}

Effects of MBCT were compared to a CONTROL condition in adults with a history of major depression and current residual

\footnotetext{
${ }^{1}$ For the per-protocol analyses (thus excluding the three participants who attended less than four MBCT sessions), results were similar to those reported above, except for one of the secondary outcomes: the association between reduction of residual symptoms and activity pleasantness. This association became nonsignificant $\left(p=.217, b^{*}=.03,95 \%\right.$ CI $[-.02$, $.08])$
} 


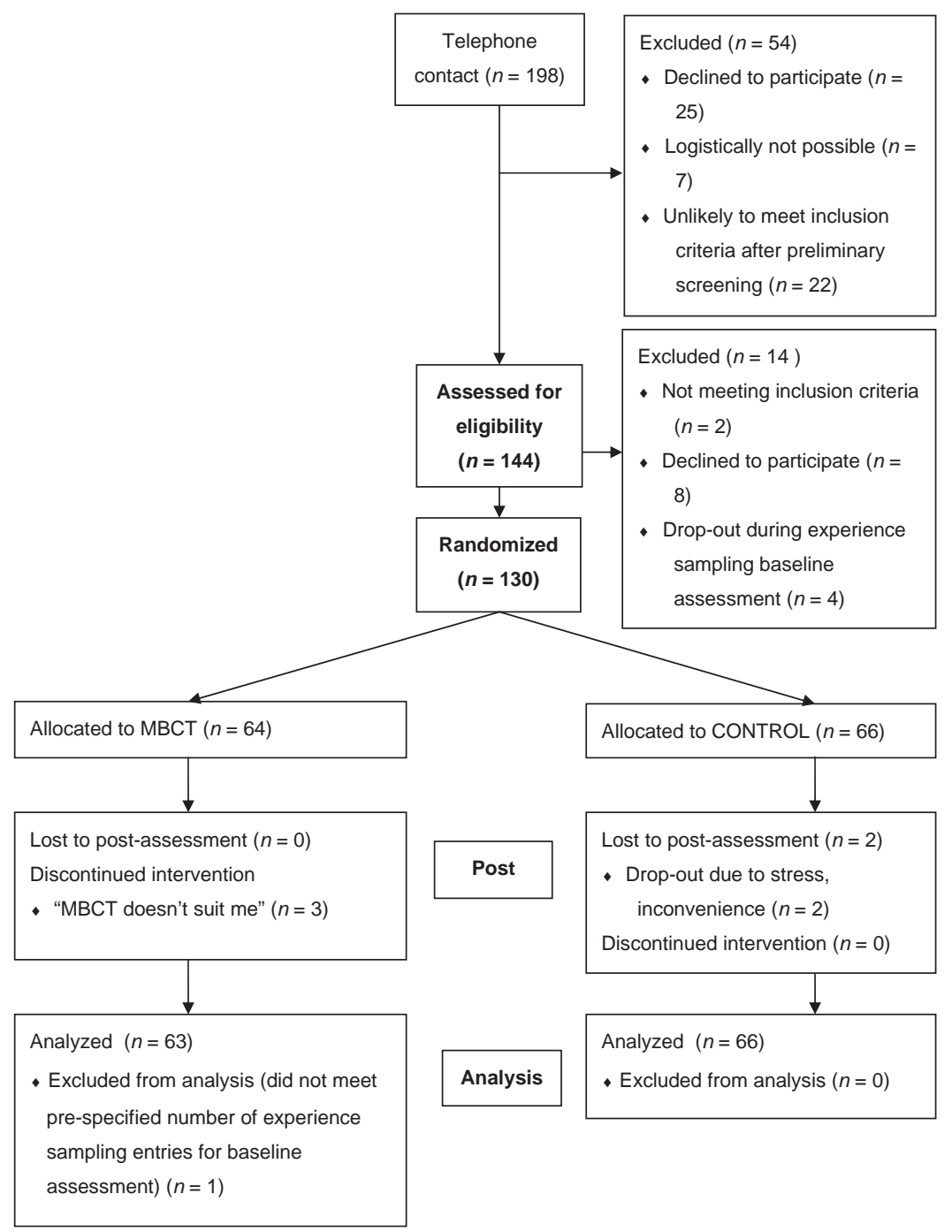

Figure 1. Participant flow diagram. MBCT $=$ mindfulness-based cognitive therapy; $\mathrm{CONTROL}=$ waitlist control condition.

symptoms. Overall, our results support the primary hypothesis that MBCT is associated with both enhanced experience of pleasant daily-life situations and improved PA responsiveness to pleasant daily-life situations. Results show that MBCT was associated with (a) more overall PA; (b) higher appraisal of activities as pleasant; and (c) higher levels of reward experience, compared to baseline, and compared to CONTROL. Special attention was given to the question of whether PA-related changes after MBCT may have simply been epiphenomena of reduced depression. The effects of treatment condition remained similar in analyses additionally correcting for reduction of depressive symptoms, indicating that MBCT affected changes in PA-related variables independent of degree of improvement in depressive symptomatology. Further analyses examined to which extent effects of MBCT on PA related variables were independent of changes in worry, rumination (variables on which MBCT research has focused so far), and NA. Effect sizes of MBCT on PA-related variables were reduced but remained significant when changes in worrying, rumination, and NA were included.
In line with our secondary hypothesis, increases in PA variables within the MBCT group were associated with reductions in residual depressive symptoms, indicating the potential relevance of increased PA for the prevention of depression. There was a doseresponse relation between reductions on the clinician-rated HDRS and the PA-related variables. MBCT participants with the highest reduction in residual symptoms (based on a tertile split of the distribution) experienced significantly stronger increases in PA, activity pleasantness, and reward experience than those with medium improvement, who in turn experienced stronger increases than those in the low improvement group (except for activity pleasantness and reward experience, where only the high improvement group differed from the other two groups).

\section{The Other Face of MBCT: Changing the Experience of Positive Emotions}

Our findings suggest that modification of a resilience phenotype (more frequent experience of daily-life PA as well as enhanced PA 

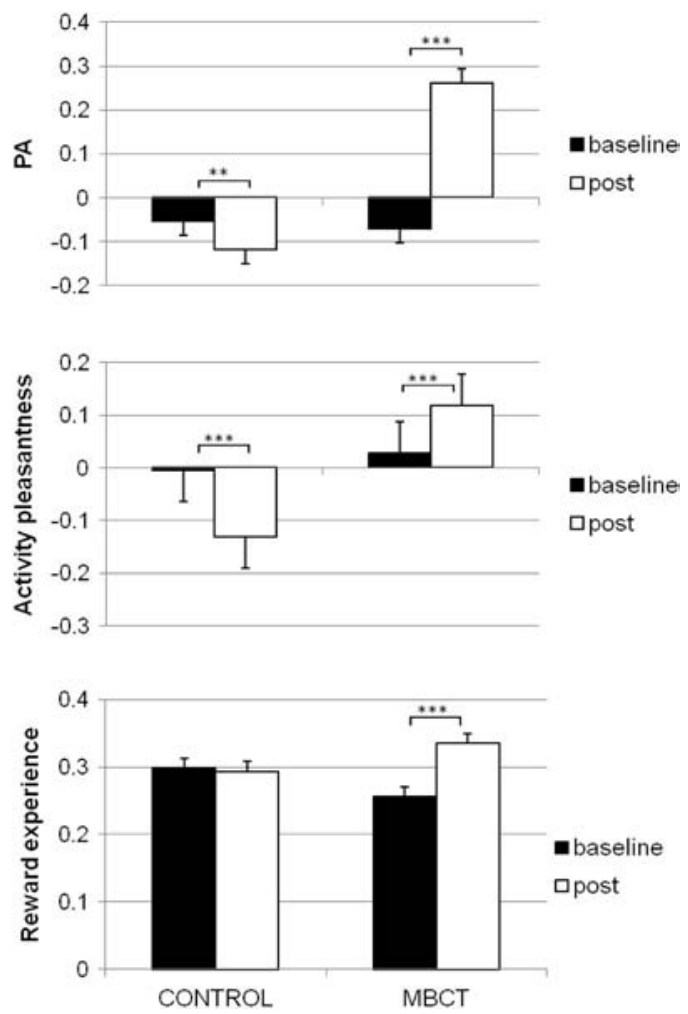

Figure 2. Standardized predicted values of positive affect (PA), activity pleasantness, and reward experience $( \pm S E)$ at baseline and postassessment in the waitlist control (CONTROL) and mindfulness-based cognitive therapy (MBCT) groups. Analyses were controlled for a person's mean baseline level on the dependent variable (PA or activity pleasantness, respectively). ${ }^{* *} p<.01 .{ }^{* * * *} p<.001$.

responsiveness to pleasant daily-life activities) is possible. Increases in PA, activity appraisal, and reward experience were specific to MBCT (with no significant increases in the control condition) and were partially independent of more commonly observed decreases in worry, rumination, and NA (Raes et al., 2009; Teasdale et al., 1995, 2000). This points to the possibility that MBCT may directly facilitate the experience of positive emotions, as suggested by Garland et al. (2010). For example, enhanced engagement with the present experience (one of the main goals of MBCT) is associated with broadening of attention (Lutz, Slagter, Dunne, \& Davidson, 2008) and may therefore lead to higher awareness of pleasant situations (which are more easily overlooked than unpleasant situations; Baumeister et al., 2001). This higher awareness of pleasant situations, in combination with the curious and mild attitude taught during MBCT, may in turn lead to increases in individuals' hedonic capacity (Schroevers \& Brandsma, 2010).

Although the current study provides initial support for the assumption that MBCT may increase the experience of reward and positive emotions (phenotypes that have been associated with better resilience against depressive symptomatology), future research should examine whether or not the experimental modification of reward experience represents a mechanism of change of MBCT. Kazdin (2007) provides a compelling account about how research on mindfulness mechanisms may best progress. For example, an association between the extent of the experimental increase in reward experience and a more favorable future course of illness would support the hypothesis of reward experience as a mechanism of change of MBCT. Studies might also investigate whether increased engagement with the present experience (as proposed above) or rather changes in third variables drive greater appreciation of, and enhanced responsiveness to, pleasant activities. Furthermore, without an active treatment comparison, it is impossible to know whether the changes in PA are specifically due to increased mindfulness. Therefore, future studies investigating associations between MBCT and change in PA should include a third attention-control arm (or alternatively a "pure" arm, in which nourishing or rewarding activities are not at all addressed during the intervention). Finally, research investigating whether certain individual differences or clinical characteristics determine individual variation in the primary changes after MBCT would also be valuable. For example, some participants may benefit from MBCT mainly through reduction of worrying, whereas others may benefit mainly through changes in the experience of positive emotions.
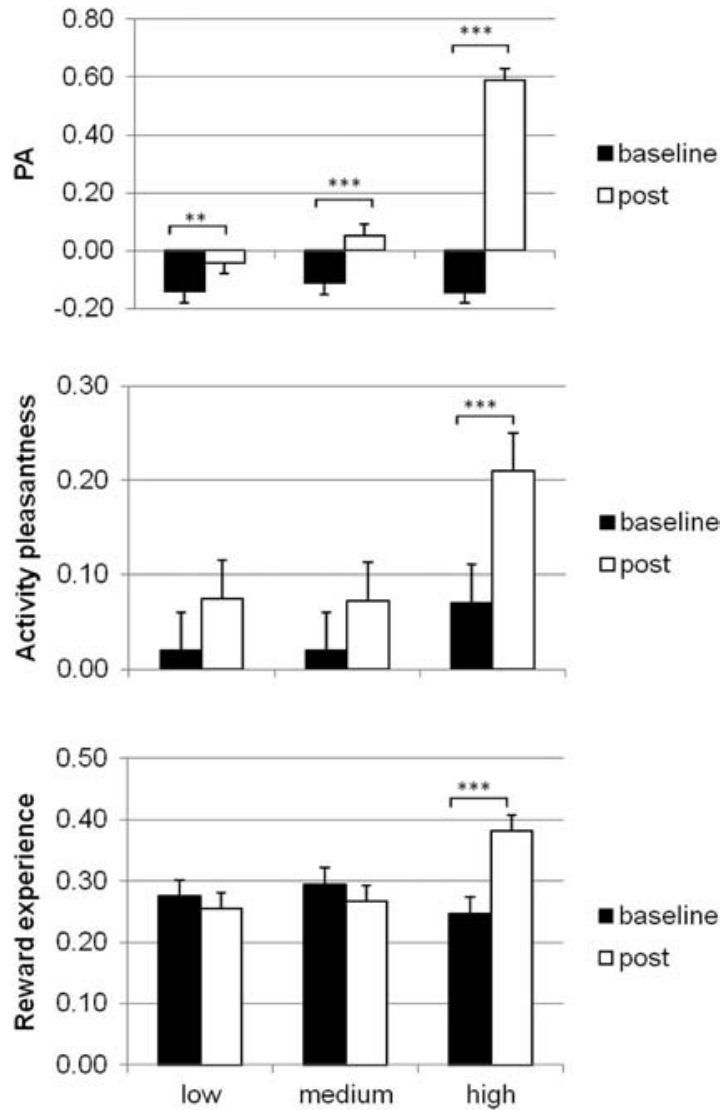

Figure 3. Association between reduction of depressive symptoms and positive affect (PA), activity pleasantness, and reward experience, before and after mindfulness-based cognitive therapy (MBCT). Analyses were controlled for a person's mean baseline level on the dependent variable (PA or activity pleasantness, respectively). Bars represent standardized predicted values $( \pm S E)$ in low, medium, and high responders (MBCT group only). Differences between baseline and postassessment increased with clinical response. ${ }^{* * *} p<.01$. $^{* * *} p<.001$. 


\section{Clinical Implications}

A growing body of literature indicates that the efficient generation of positive emotions may be a resilience phenotype: PA is very important for well-being in general (Folkman \& Moskowitz, 2000; Seligman et al., 2005) and the prevention of and recovery from depression in particular (Garland et al., 2010; Wichers et al., 2009). Several studies suggest that it would be clinically relevant to change the ability to experience of PA if that was possible. High levels of reward experience have been shown to protect against the development of affective symptoms (Geschwind et al., 2010; Wichers et al., 2010) and were associated with recovery from depression after pharmacotherapy (Wichers et al., 2009) in other, unrelated samples. The present study, to our knowledge, is the first to show that a behavioral, nonpharmacological intervention is associated with enhanced generation of PA during pleasant dailylife activities. However, due to the lack of an active intervention group, we cannot assume causality. An alternative explanation for our findings is that decreases in depressive symptoms could lead to increases in PA.

Despite statistical significance, the clinical significance may seem less obvious, because increases in PA are relatively small (e.g., PA increased from 3.7 to 4.2 on a 7-point Likert scale in the MBCT condition). On the other hand, a previous study showed that very small increases in PA can be strong predictors of clinically relevant change. For example, small improvements in dailylife PA (from 3.3 to 3.6 on a 7-point Likert scale) during the first week of antidepressant treatment were associated with a 34 times higher chance on achieving remission 6 weeks later, compared to no change or worsening of PA during the first week (Geschwind et al., 2011).

Another clinically relevant finding is that increased reward experience following MBCT co-occurred with the reduction of residual complaints (which are harmful and increase the risk for future episodes of major depression; Judd et al., 1998). Effects of MBCT on PA-related variables did not change when corrected for change in residual depressive symptoms, indicating that PArelated improvements were not simply epiphenomena of reductions in depressive symptoms. To verify this claim more definitely, however, replication of results in a symptom-free sample is desirable. Furthermore, future studies should look beyond changes in traditionally researched $\mathrm{MBCT}$ outcome variables and investigate to what extent MBCT-induced changes in positive emotionality and other resilience-related constructs contribute to recovery from depression and relapse prevention.

\section{Strengths and Limitations}

Strengths of the current study include the use of the ESM to measure changes in positive affectivity. ESM repeatedly measures positive emotions as they occur in daily life, thus increasing ecological validity and minimizing memory bias (Csikszentmihalyi \& Larson, 1987). Although ESM relies on self-report, demand bias for the main outcome (change in PA-related variables) was unlikely for the following reasons: (a) PA items were hidden among other items describing the current activity and situation, and participants were blind to the hypotheses; (b) MBCT's main focus is to encourage participants to engage with the present moment (although MBCT does also address nourishing activities); and (c) most previous research has focused on negative emotions, rumination, or worry. Another strength of the current study is that inclusion criteria were intentionally kept at a low threshold to enhance generalizability. Moreover, attrition rate was low, and the main analyses focused on the intention-to-treat sample, thus analyzing all participants randomized to treatment. Finally, the study was a randomized controlled trial, thus reducing selection bias, and there were no prior differences in baseline or clinical characteristics between MBCT and CONTROL.

Limitations are (a) that the current trial did not include an active control intervention arm, making conclusions about active treatment ingredients impossible; (b) the all-Caucasian sample; and (c) the absence of objective information on competent treatment delivery and adherence to the MBCT protocol. Another limitation is (d) the reliance on self-reported entry completion time for distinguishing valid from invalid experience sampling reports. However, an earlier study comparing self-reported completion time and objective completion time (obtained through monitoring by hidden electronic devices; Jacobs et al., 2005) indicates that self-reported completion times are reliable. Furthermore, (e) both participants and assessors were not blind to treatment condition due to pragmatic limitations. Importantly though, participants and assessors were unaware of direction and extent of change in PA-related variables (the main trial outcome) when assessing residual depressive symptoms. Also, associations between increases in PA-related variables and decreases in residual depressive symptoms were unlikely to be due to biased assessment, because results of selfreport (IDS-SR) and interview-based (HDRS) assessments of residual depressive symptoms corresponded. A further limitation (f) is that it is impossible to say whether increase in activity appraisal indeed represents a tendency to appraise similar activities as more pleasant than before MBCT or rather a tendency to engage in more pleasant activities. However, either option has clinical relevance. The choice not to have external observers rate pleasantness of activities was made consciously, because the experience of everyday activities is entirely subjective. Moreover, this was a withinsubject design, and participants were compared to themselves. Finally, (g) the low-threshold inclusion criteria generate more heterogeneity between participants. However, there were no significant differences on any key variables between MBCT and CONTROL groups at baseline. Furthermore, the low-threshold inclusion criteria warrant generalizability to most individuals with residual depressive symptoms (though replication in a symptomfree sample would be desirable).

\section{Conclusion}

This is the first study to show that a behavioral intervention can lead to increased experience of momentary positive emotions as well as greater appreciation of, and enhanced responsiveness to, pleasant daily-life activities. From a clinical point of view, this is a very relevant finding because it means that a nonpharmacological intervention may potentially improve the reward system. The clinical impact is considerable, because it makes prevention efforts targeting reward experience in vulnerable groups more acceptable.

More research is needed with regard to active treatment ingredients, mechanisms of change, and the degree to which positive emotions and other resilience-related phenotypes contribute to recovery from depression and prevention of relapse or recurrence. 


\section{References}

Baer, R. A. (2003). Mindfulness training as a clinical intervention: A conceptual and empirical review. Clinical Psychology: Science and Practice, 10, 125-143. doi:10.1093/clipsy.bpg015

Bagby, R. M., Ryder, A. G., Schuller, D. R., \& Marshall, M. B. (2004). The Hamilton Depression Rating Scale: Has the gold standard become a lead weight? The American Journal of Psychiatry, 161, 2163-2177. doi: 10.1176/appi.ajp.161.12.2163

Barge-Schaapveld, D. Q. C. M., Nicolson, N. A., Berkhof, J., \& deVries, M. W. (1999). Quality of life in depression: Daily life determinants and variability. Psychiatry Research, 88, 173-189. doi:10.1016/S01651781(99)00081-5

Baumeister, R. F., Bratslavsky, E., Finkenauer, C., \& Vohs, K. D. (2001). Bad is stronger than good. Review of General Psychology, 5, 323-370. doi:10.1037/1089-2680.5.4.323

Beck, A. T., Rush, A. J., Shaw, B. F., \& Emergy, G. (1979). Cognitive therapy of depression. New York, NY: Guilford Press.

Bondolfi, G., Jermann, F., Van der Linden, M., Gex-Fabry, M., Bizzini, L., Rouget, B. W., . . . Bertschy, G. (2010). Depression relapse prophylaxis with mindfulness-based cognitive therapy: Replication and extension in the Swiss health care system. Journal of Affective Disorders, 122, 224-231. doi:10.1016/j.jad.2009.07.007

Brown, K. W., \& Ryan, R. M. (2003). The benefits of being present: Mindfulness and its role in psychological well-being. Journal of Personality and Social Psychology, 84, 822-848. doi:10.1037/00223514.84.4.822

Bylsma, L. M., Morris, B. H., \& Rottenberg, J. (2008). A meta-analysis of emotional reactivity in major depressive disorder. Clinical Psychology Review, 28, 676-691. doi:10.1016/j.cpr.2007.10.001

Bylsma, L. M., Taylor-Clift, A., \& Rottenberg, J. (2011). Emotional reactivity to daily events in major and minor depression. Journal of Abnormal Psychology, 120, 155-167. doi:10.1037/a0021662

Clayton, D., \& Hill, M. (1993). Wald tests. In D. Clayton \& M. Hills (Eds.), Statistical models in epidemiology (pp. 101-102). Oxford, England: Oxford Science.

Cohn, M. A., Fredrickson, B. L., Brown, S. L., Mikels, J. A., \& Conway, A. M. (2009). Happiness unpacked: Positive emotions increase life satisfaction by building resilience. Emotion, 9, 361-368. doi:10.1037/ a0015952

Conway, M., Csank, P. A. R., Holm, S. L., \& Blake, C. K. (2000). On assessing individual differences in rumination on sadness. Journal of Personality Assessment, 75, 404-425. doi:10.1207/S15327752JPA7503_04

Csikszentmihalyi, M., \& Larson, R. (1987). Validity and reliability of the Experience Sampling Method. Journal of Nervous and Mental Disease, 175, 526-536. doi:10.1097/00005053-198709000-00004

Delespaul, P. A. E. G. (1995). Assessing schizophrenia in daily life: The Experience Sampling Method. Maastricht, the Netherlands: Maastricht University Press.

Easterlin, B., \& Cardena, E. (1998-1999). Cognitive and emotional differences between short and long term vipassana meditators. Imagination, Cognition and Personality, 18, 69-81.

Evans, S., Ferrando, S., Findler, M., Stowell, C., Smart, C., \& Haglin, D. (2008). Mindfulness-based cognitive therapy for generalized anxiety disorder. Journal of Anxiety Disorders, 22, 716-721. doi:10.1016/ j.janxdis.2007.07.005

First, M. B., Spitzer, R. L., Gibbon, M., \& Williams, J. B. W. (2002). Structured Clinical Interview for DSM-IV-TR Axis 1 Disorders, Research Version. New York, NY: Biometrics Research, New York State Psychiatric Institute.

Folkman, S., \& Moskowitz, J. T. (2000). Positive affect and the other side of coping. American Psychologist, 55, 647-654. doi:10.1037/0003066X.55.6.647

Fredrickson, B. L., Cohn, M. A., Coffey, K. A., Pek, J., \& Finkel, S. M. (2008). Open hearts build lives: Positive emotions, induced through loving-kindness meditation, build consequential personal resources. Journal of Personality and Social Psychology, 95, 1045-1062. doi: $10.1037 / \mathrm{a} 0013262$

Garland, E. L., Fredrickson, B. L., Kring, A. M., Johnson, D. P., Meyer, P. S., \& Penn, D. L. (2010). Upward spirals of positive emotions counter downward spirals of negativity: Insights from the broaden-and-build theory and affective neuroscience on the treatment of emotion dysfunctions and deficits in psychopathology. Clinical Psychology Review, 30, 849-864. doi:10.1016/j.cpr.2010.03.002

Geschwind, N., Nicolson, N. A., Peeters, F., van Os, J., Barge-Schaapveld, D., \& Wichers, M. (2011). Early improvement in positive rather than negative emotion predicts remission from depression after pharmacotherapy. European Neuropsychopharmacology, 21, 241-247. doi: 10.1016/j.euroneuro.2010.11.004

Geschwind, N., Peeters, F., Jacobs, N., Delespaul, P., Derom, C., Thiery, E., ... Wichers, M. (2010). Meeting risk with resilience: High daily life reward experience preserves mental health. Acta Psychiatrica Scandinavica, 122, 129-138. doi:10.1111/j.1600-0447.2009.01525.x

Hamilton, M. (1960). A rating scale for depression. Journal of Neurology, Neurosurgery \& Psychiatry, 23, 56-62. doi:10.1136/jnnp.23.1.56

Jacobs, N., Nicolson, N. A., Derom, C., Delespaul, P., van Os, J., \& Myin-Germeys, I. (2005). Electronic monitoring of salivary cortisol sampling compliance in daily life. Life Sciences, 76, 2431-2443. doi: 10.1016/j.lfs.2004.10.045

Judd, L. L., Akiskal, H. S., Maser, J. D., Zeller, P. J., Endicott, J., Coryell, W., ... Keller, M. (1998). Major depressive disorder: A prospective study of residual subthreshold depressive symptoms as predictor of rapid relapse. Journal of Affective Disorders, 50, 97-108. doi:10.1016/S01650327(98)00138-4

Kabat-Zinn, J. (1990). Full catastrophe living: The program of the Stress Reduction Clinic at the University of Massachusetts Medical Center. New York, NY: Dell.

Kazdin, A. E. (2007). Mediators and mechanisms of change in psychotherapy research. Annual Review of Clinical Psychology, 3, 1-27. doi 10.1146/annurev.clinpsy.3.022806.091432

Killingsworth, M. A., \& Gilbert, D. T. (2010, November 12). A wandering mind is an unhappy mind. Science, 330, 932. doi:10.1126/science .1192439

Kuyken, W., Byford, S., Taylor, R. S., Watkins, E., Holden, E., White, K., ... Teasdale, J. D. (2008). Mindfulness-based cognitive therapy to prevent relapse in recurrent depression. Journal of Consulting and Clinical Psychology, 76, 966-978. doi:10.1037/a0013786

Kuyken, W., Watkins, E., Holden, E., White, K., Taylor, R. S., Byford, S., ... Dalgleish, T. (2010). How does mindfulness-based cognitive therapy work? Behaviour Research and Therapy, 48, 1105-1112. doi:10.1016/ j.brat.2010.08.003

Lutz, A., Slagter, H. A., Dunne, J. D., \& Davidson, R. J. (2008). Attention regulation and monitoring in meditation. Trends in Cognitive Sciences, 12, 163-169. doi:10.1016/j.tics.2008.01.005

Lyubomirsky, S., King, L., \& Diener, E. (2005). The benefits of frequent positive affect: Does happiness lead to success? Psychological Bulletin, 131, 803-855. doi:10.1037/0033-2909.131.6.803

Ma, S. H., \& Teasdale, J. D. (2004). Mindfulness-based cognitive therapy for depression: Replication and exploration of differential relapse prevention effects. Journal of Consulting and Clinical Psychology, 72, 31-40. doi:10.1037/0022-006X.72.1.31

Meyer, T. J., Miller, M. L., Metzger, R. L., \& Borkovec, T. D. (1990). Development and validation of the Penn State Worry Questionnaire. Behaviour Research and Therapy, 28, 487-495. doi:10.1016/00057967(90)90135-6

Morris, B. H., Bylsma, L. M., \& Rottenberg, J. (2009). Does emotion predict the course of major depressive disorder? A review of prospective studies. British Journal of Clinical Psychology, 48, 255-273. doi: 10.1348/014466508X396549 
Mueller, T. I., Leon, A., Keller, M., Solomon, D., Endicott, J., Coryell, W., ... Maser, J. D. (1999). Recurrence after recovery from major depressive disorder during 15 years of observational follow-up. The American Journal of Psychiatry, 156, 1000-1006.

Myin-Germeys, I., \& van Os, J. (2007). Stress-reactivity in psychosis: Evidence for an affective pathway to psychosis. Clinical Psychology Review, 27, 409-424. doi:10.1016/j.cpr.2006.09.005

Myin-Germeys, I., van Os, J., Schwartz, J. E., Stone, A. A., \& Delespaul, P. A. (2001). Emotional reactivity to daily life stress in psychosis. Archives of General Psychiatry, 58, 1137-1144. doi:10.1001/ archpsyc.58.12.1137

Nutt, D., Demyttenaere, K., Janka, Z., Aarre, T., Bourin, M., Canonico, P. L., . . Stahl, S. (2007). The other face of depression, reduced positive affect: The role of catecholamines in causation and cure. Journal of Psychopharmacology, 21, 461-471. doi:10.1177/0269881106069938

Peeters, F., Berkhof, J., Delespaul, P., Rottenberg, J., \& Nicolson, N. A. (2006). Diurnal mood variation in major depressive disorder. Emotion, 6, 383-391. doi:10.1037/1528-3542.6.3.383

Peeters, F., Nicolson, N. A., Berkhof, J., Delespaul, P., \& De Vries, M. (2003). Effects of daily events on mood states in major depressive disorder. Journal of Abnormal Psychology, 112, 203-211. doi:10.1037/ 0021-843X.112.2.203

Raes, F., Dewulf, D., Van Heeringen, C., \& Williams, J. M. G. (2009). Mindfulness and reduced cognitive reactivity to sad mood: Evidence from a correlational study and a non-randomized waiting list controlled study. Behaviour Research and Therapy, 47, 623-627. doi:10.1016/ j.brat.2009.03.007

Raes, F., Hermans, D., \& Eelen, P. (2003). De nederlandstalige versie van de Ruminative Response Scale (RRS-NL) en de Rumination on Sadness Scale (RSS-NL) [The Dutch version of the Ruminative Response Scale (RRS-NL) and the Rumination on Sadness Scale (RSS-NL)]. Gedragstherapie, 36, 97-104.

Rush, A. J., Gullion, C. M., Basco, M. R., Jarrett, R. B., \& Trivedi, M. H. (1996). The Inventory of Depressive Symptomatology (IDS): Psychometric properties. Psychological Medicine, 26, 477-486. doi:10.1017/ S0033291700035558

Rush, A. J., Trivedi, M. H., Wisniewski, S. R., Nierenberg, A. A., Stewart, J. W., Warden, D., ... Fava, M. (2006). Acute and longer-term outcomes in depressed outpatients requiring one or several treatment steps: A STAR*D report. The American Journal of Psychiatry, 163, 19051917. doi:10.1176/appi.ajp.163.11.1905

Schroevers, M. J., \& Brandsma, R. (2010). Is learning mindfulness associated with improved affect after mindfulness-based cognitive therapy? British Journal of Psychology, 101, 95-107. doi:10.1348/ 000712609X424195
Segal, Z. V., Williams, J. M. G., \& Teasdale, J. D. (2002). Mindfulnessbased cognitive therapy for depression: A new approach to preventing relapse. New York, NY: Guilford Press.

Seligman, M. E. P., Steen, T. A., \& Peterson, C. (2005). Positive psychology progress: Empirical validation of interventions. American Psychologist, 60, 410-421. doi:10.1037/0003-066X.60.5.410

Snijders, T., \& Bosker, R. (1999). Multilevel analysis: An introduction to basis and advanced multilevel modeling. London, England: Sage.

StataCorp. (2009). Stata statistical software: Release 11 [Computer software]. College Station, TX: Author.

Teasdale, J. D., Segal, Z. V., \& Williams, J. M. G. (1995). How does cognitive therapy prevent depressive relapse and why should attentional control (mindfulness) training help? Behaviour Research and Therapy, 33, 25-39. doi:10.1016/0005-7967(94)E0011-7

Teasdale, J. D., Segal, Z. V., Williams, J. M. G., Ridgeway, V. A., Soulsby, J. M., \& Lau, M. A. (2000). Prevention of relapse/recurrence in major depression by mindfulness-based cognitive therapy. Journal of Consulting and Clinical Psychology, 68, 615-623. doi:10.1037/0022006X.68.4.615

Watson, D., Clark, L. A., \& Carey, G. (1988). Positive and negative affectivity and their relation to anxiety and depressive disorders. Journal of Abnormal Psychology, 97, 346-353. doi:10.1037/0021-843X 97.3.346

Wichers, M. C., Barge-Schaapveld, D. Q. C. M., Nicolson, N. A., Peeters, F., de Vries, M., Mengelers, R., \& van Os, J. (2009). Reduced stresssensitivity or increased reward experience: The psychological mechanism of response to antidepressant medication. Neuropsychopharmacology, 34, 923-931. doi:10.1038/npp.2008.66

Wichers, M., Peeters, F., Geschwind, N., Jacobs, N., Simons, C. J. P., Derom, C., ... van Os, J. (2010). Unveiling patterns of affective responses in daily life may improve outcome prediction in depression: A momentary assessment study. Journal of Affective Disorders, 124, 191195. doi:10.1016/j.jad.2009.11.010

Williams, J. M. G. (2008). Mindfulness, depression and modes of mind. Cognitive Therapy and Research, 32, 721-733. doi:10.1007/s10608008-9204-z

Zautra, A. J., Johnson, L. M., \& Davis, M. C. (2005). Positive affect as a source of resilience for women in chronic pain. Journal of Consulting and Clinical Psychology, 73, 212-220. doi:10.1037/0022-006X .73.2.212

Received November 29, 2010

Revision received May 11, 2011 Accepted June 2, 2011 